\title{
Management of personality disorders in acute in-patient settings. Part 1: Borderline personality disorders
}

\author{
Leonard Fagin
}

\begin{abstract}
General principles of management of patients with personality disorders admitted in crises to the inpatient unit are discussed. The role of the acute ward in the overall plan of care, the clinical thresholds to consider in deciding whether admission is appropriate and the main elements of the in-patient care plan are outlined. The management of patients with borderline personality disorder, who constitute the majority of such admissions, is discussed in detail.
\end{abstract}

This is the first of two articles that tackle the management problems presented when patients with personality disorders are admitted in crisis to the in-patient unit. The second article (Fagin, 2004, this issue) discusses less-common personality disorders.

The management and treatment of personality disorders have caused considerable controversy among psychiatrists in the UK. In a well-reasoned article in a past issue of this journal, Gwen Adshead outlined the main issues relating to the status of personality disorders as nosological entities and their treatability (Adshead, 2001). Debates held at the Royal College of Psychiatrists' annual meetings and in its regional divisions indicate a major split in the profession, with a strong lobby suggesting that people with personality disorders should, in the current state of knowledge, be considered either not treatable at all or, perhaps more emolliently, treatable only in specialised settings. This view is supported by the scarcity of reliable and robust treatment data that offer reasonable assurance of good outcomes. The debate about dangerous severe personality disorder, the possibility of such a diagnosis being amenable to anticipatory compulsory detention under a revised Mental Health Act and the creation of multi-agency public protection panels has polarised views even further. Recent guidance on personality disorders from the National Institute of Mental Health in England has helpfully raised many issues relating to this often-excluded group, which prevalence studies suggest constitutes $10-13 \%$ of the population (National Institute of Mental Health in England, 2003). My criticism of this report is that it tends to play down the role of the acute in-patient setting in the lifetime experiences of people with personality disorders.

While this debate continues, most clinicians in adult psychiatry have to face the fact that, like it or not, they may have to manage people with personality disorders in acute settings at some time, either because of crises that lead to emergency admissions, or because Axis II personality disorders complicate other presentations, such as depression and schizophrenia, and require complex handling if the basic issues are to be appropriately tackled (Norton \& Hinshelwood, 1996; Mulder, 2002).

The aim of my two articles is not to suggest that personality disorders are treatable in acute inpatient settings, but to look at the resources available in those environments that can respond to the extremes of personality difficulties, whether patients are admitted with this sole diagnosis in a state of crisis, or whether personality is an added dimension of another psychiatric diagnosis. My approach is to look at the possibility of acute clinical management strategies in certain subtypes of personality disorder and, if favourable circumstances allow, to extend beyond this, incorporating a longer-term outlook. This model of care has recently shown some promising results (Chiesa et al, 2002). Although I agree that acute in-patient units are generally unsuitable for long-term work with people with personality disorders, I concur with Norton \& Hinshelwood (1996) when they state that 'an admission ..., although often problematic, can be conceived as an opportunity'. In all of these

Leonard Fagin is a consultant psychiatrist and the Clinical Director of the North East London Mental Health NHS Trust. He also holds the post of honorary senior lecturer at University College London (South Forest Centre, 21 Thorne Close, Leytonstone, London E11 4HU, UK). Among his research interests he has studied the psychological effects of unemployment, occupational stress, standards of care in in-patient units and the effects of deinstitutionalisation. 
presentations, the question for staff is whether the patient can embark on meaningful change to their distorted behavioural and emotional patterns in an appropriate therapeutic environment. For this to take place, staff will have to enlarge their skills base and be supported and supervised by local specialist teams dedicated to the assessment, management and treatment of personality disorder (National Institute of Mental Health in England, 2003).

\section{Management principles}

Bateman \& Tyrer (Bateman \& Tyrer, 2002; National Institute for Mental Health in England, 2003, p. 27) and Davison (2002) have identified the guiding principles of effective therapy for people with personality disorders, and aspects of these can be taken into account when we envisage the role of the acute ward in the management of care (Box 1 ).

One of the controversial points in this area is the clinical thresholds that need to be set as indicators for in-patient admissions (Bateman \& Tyrer, cited in National Institute for Mental Health in England, 2003), particularly taking into account the possible deleterious effect on the patient or others of such an intervention (Box 2).

Translated to an in-patient service, the main elements of psychiatric interventions are listed in Box 3 .

Part of the problem created by patients with Axis II disorders is that they are, on the whole, very unpopular with staff, and therefore do not contribute to the creation of a therapeutic alliance. The switch from the role of victim to that of perpetrator provokes equally extreme responses from staff, from 'kind defender' to 'cruel attacker' (Norton \& Dolan, 1995). Not everybody can work with patients with personality disorders. Duggan (2002) has identified some of the key competencies of staff that help them work effectively with such patients. These include emotional resilience, clarity about personal and interpersonal boundaries, and ability to tolerate the intense emotional impact that these patients can have on them. Added to this is the need for effective and regular supervision, at both individual and team level.

In this first article I confine myself to making a few clinical suggestions on the most common of the personality disorders likely to present in acute settings, borderline personality. I do not broach the subject of how personality issues can be dealt with when they complicate another Axis I diagnosis, although I believe that many of the points are equally valid in these circumstances. In Part 2, I focus on personality disorders that, although less frequently presenting in acute settings, still pose management difficulties.
Box 1 Management principles in the treatment of personality disorders (after Bateman \& Tyrer, 2002)

Staff should devote effort to achieving adherence to the treatment, which should:

- be well structured

- have a clear focus

- have a theoretical basis that is coherent to both staff and patient

- be relatively long-term

- be well integrated with other services available to the patient, using the care programme approach as a main means of networking, communicating and reviewing plans between different elements of the service

- involve a clear treatment alliance between staff and patient

\section{Borderline personality disorder}

This group, consisting predominantly of female patients, are likely to be admitted in a crisis to an inpatient environment, usually with suicidal or selfharming intent. Although the diagnosis of borderline personality disorder is controversial and still being argued about, the combination of features of impulsive and sometimes manipulative selfdestructive behaviour, pathological attachments tinged with dread of abandonment, unstable selfimage, affective lability, transient circumscribed psychotic-like or magical thinking, as well as overwhelming angry demands and feelings of entitlement, is not only clinically recognisable but makes these patients most likely to present after hours to a busy, and probably overwhelmed, junior

Box 2 Indicators for admission of patients with a personality disorder

- Crisis intervention, particularly to reduce risk of suicide or violence to others

- Comorbid psychiatric disorder such as depression or a brief psychotic episode

- Chaotic behaviour endangering the patient and the treatment alliance

- To stabilise existing medication regimens

- Review of the diagnosis and the treatment plan

- Full risk assessment

- Above all, the unit must have the capacity, in terms of skills, staffing and clinical pressures, to manage the admission 
Box 3 The main elements of psychiatric intervention in an acute in-patient setting

- Informality

- Careful assessments by experienced staff, focusing on the present crisis and need for containment

- Involvement of significant others, carers, relatives and other agencies in the assessment

- Early care plan with specified goals agreed and communicated to all staff and the patient, paying special attention to perceived or real inconsistencies. Anticipation of crises, especially about impulsive discharge, selfharm, drug use, sexual promiscuity or aggression, and establishment an agreed multidisciplinary response

- A focus on immediate needs, mostly of a practical nature

- Clear boundaries regarding tolerable behaviour, including aggression, suicidal gestures, use of illicit substances or alcohol and absconsions

- Effective use of in-patient groups

- Treatment of psychiatric symptoms with medication when necessary

- Staff support groups and supervision looking at countertransference reactions, particularly of junior staff, who may become overinvolved

- Early discharge arrangements when crisis has been overcome

- Readiness to discharge if goals are not met

- Referral to community or specialist services on discharge, with close and careful handovers through the CPA

- Short duration of admissions

- Consideration of comorbidity of a personality disorder if treatment for an Axis I disorder does not meet with the predictable response

doctor on-call. The fears of what might happen if these patients are not contained inevitably lead to admission. Their histories typically reveal parental rejection and abandonment as well as sexual abuse at an early stage of development, with recent incidents of assault or neglect as triggering factors. Those supporting a biogenetic model have also observed a preponderance of attention-deficit disorders, learning disabilities and conduct disorders in the histories of these patients.

Patients with borderline personality disorder provoke the most extreme countertransferential feelings in staff, who report that they feel abused and deceived. The tendency of these patients to either deify or demonise their carers, dependent on whether their urgent and immediate needs are gratified or not, places staff in very difficult and invidious dilemmas. In the same vein, these patients may appear to be in the depth of depression and despair one minute, and the next may be playing games and laughing with their fellow patients as if nothing has happened. The difficulties imposed by these patients when they are suicidal are particularly troublesome (Matsberger, 1999), including accusations that staff do not care enough, are insensitive, have lost hope or secretly wish them to die. In a culture of risk management and serious-incident investigations, this may be difficult to bear. It can cause staff to feel that they carry more of the responsibility for keeping these suicidal patients alive than do the patients themselves, a condition which Hendin (1981) described as coercive bondage. The catch-22 is that analysis of completed suicides by patients with borderline personality disorder has shown that perceived rejection by caregivers has often been the precipitating cause (Kullgren, 1988).

These negative features should be put into context. Studies of the natural course of this illness are rare, but analyses of data of patients in contact with psychiatric services report generally good outcomes over prolonged periods of observation, if their impulsive behaviour at the peak of presentation does not prematurely lead to a tragic end, which, considering the frequency of suicidal threat, is not that common (Stone, 1990).

As with all other groups of personality disorders, there are no quick and easy answers in borderline personality disorder. Although some researchers have reported considerable improvements in specialised units run on psychodynamic (Bateman \& Fonagy, 1999) or cognitive-behavioural or dialectical behavioural lines (Linehan et al, 1991; Gunderson, 2001), these interventions are not easily available in all districts.

\section{The role of the acute in-patient setting}

Reported clinical experience indicates (and here I have found excellent supporting articles in the nursing journals) that there is a role for the in-patient unit where patients are often taken in an emergency, if a graded and well-informed response can be agreed between staff and patient. The approach, however, requires good team functioning and clarity of purpose, as without these, team difficulties are likely to be exposed with these patients. Good leadership is also required, and in particular a good understanding of purpose between the senior nurse and the consultant psychiatrist (Piccinino, 1990). The aim is to restore a sense of responsibility and rely on the patient's previously good internal resources (Wester, 1991). These admissions should not be 
prolonged (Nehls, 1994a,b), mostly dealing with the emergent problems that have precipitated the crisis. Patterns of impulsive responses and wild fluctuations in attitudes and attachments to and from staff can be witnessed and discussed, and once the emotional crisis subsides can be worked through in focused individual and group therapeutic interventions, with an emphasis on problem-solving. A major source of argument is the nature of response to suicidal and self-harming behaviour. Clear understanding of the meaning of that behaviour and the aims of the staff's response can help patients in a number of ways, for example by enabling them

- to improve their interpersonal skills during conflicts

- to increase their internal regulation of unwanted emotions

- to develop the skills to tolerate emotional distress until change occurs

- to learn self-management (Gallop, 1992).

A difficult balance needs to be arrived at between ensuring patients' safety by observation, and the avoidance of collusion with helplessness and abdication of responsibility for the patient's own behaviour (Sederer \& Thornbeck, 1986). These patients are likely to cause major rifts in the team, splitting it into those who believe the patient to be manipulative and in need of firm, controlling interventions, and those who become overprotective and tolerant beyond reasonable limits (Kaplan, 1986; O'Brien, 1998). The importance of staff discussions and support groups in these circumstances cannot be overstressed. Staff have to be prepared for regular readmissions, and it is helpful to keep a historical perspective, looking at changes over time, as this can give hope when they feel despondent about possible eventual change when all they see is repeated similar presentations.

\section{Discharge planning}

In all of this, we need to remember that, on discharge, these patients are likely also to be a concern for community clinicians, and that effective communication and sharing of ideas should be established between in-patient and community teams. Likewise, family members are likely to be enmeshed and entangled in the presentation and management of the patient, and sometimes contribute to splitting or undermining of coherent staff approaches. In both these scenarios, the care programme approach (CPA) meeting, where clear boundaries, expectations and responsibilities, as well as contingency planning, are discussed, can become a very useful tool.

As noted above, a major hurdle to be overcome in the management of these patients is the hopelessness they provoke by repeated acting out and incessant demands, crossing of boundaries and weak therapeutic alliances. This leads many patients to sabotage well-prepared care plans and to present only in states of major upheaval. In this situation, the in-patient ward represents the only constancy, a safe place able to contain and hold, even if this creates an ambiguous and sometimes intolerable attachment. This contrasts with the experience of most people with borderline personality disorder, who have had tenuous parental relationships that often end in their being abandoned to their own devices. They are in fact 'soothed' and supported by the notion that the safety net of the ward is available. Slowly, over time, many patients are able to take in and appreciate this approach, and to some extent take over the soothing role without being precipitated into a crisis.

Another point to bear in mind is that patients with borderline personality disorder form a very heterogeneous group, and that set and inflexible responses will not be always appropriate. Sometimes patients just need to be held, at other times supported. On occasions where risk-taking behaviour is predominant, boundaries need to be firmly clarified and adhered to (Rouse, 1994). Staff will have to intuit when patients are ready to benefit from some degree of confrontational and interpretive techniques. Before these can be attempted, patients need to feel that their internal feelings are empathically validated, particularly if recent events open up old wounds relating to early childhood traumas.

\section{Management principles}

Box 4 summarises several of the principles of technique suggested by Gabbard (2000) that broadly apply to most patients with borderline personality disorder. These principles are enlarged on in Box 5 .

Box 4 Principles of management of patients with borderline personality disorder in inpatient units

- Maintain flexibility

- Establish conditions to make the patient safe

- Tolerate intense anger, aggression and hate

- Promote reflection

- Set necessary limits

- Establish and maintain the therapeutic alliance

- Avoid splitting between psychotherapy and pharmacotherapy (see Box 5)

- Avoid or understand splitting between different members of staff, either in hospital or in the community

- Monitor countertransference feelings 
I have already indicated the difficulties that patients with borderline personality disorder pose for staff in in-patient environments. A not uncommon scenario is where patients perceive themselves to be 'special' and different from others, and therefore requiring extra attention. At other times they may form a clique with other like-minded individuals, and purposefully disrupt the therapeutic milieu by sabotaging collaborative activities, undermining staff or disparaging their work in the unit. Others simply display passive resistance and go into monumental sulks. Overall, the treatment plan aims to avoid regression and support the contention that patients can eventually restore their adaptive functions and control chaotic, selfdestructive urges. Medication is used only to help people take back control over their feelings. The structure of the hospital day implies the rediscovery of order and a predictable pattern of interactive opportunities with individual staff and groups. The focus is on the understanding of the precipitants of the crisis, the pattern of responses and how these link up with previous traumatic experiences. When the furore has died down, patients may be able to look at alternative ways of responding, accept the consequences of their actions and validate remaining ego strengths and abilities. When setting limits, staff must be able to say 'no' without malice, explaining that this is in the interests of the patient and not, as patients often construe it, a sadistic means of control. If there are attempts at self-harm such as wrist-slashing, staff may need to convey

\section{Box 5 Principles of management - an elaboration}

Maintain flexibility

Establish conditions that keep the patient safe

Tolerate intense anger, aggression and hate

Promote reflection

Set necessary limits

Establish and maintain the therapeutic alliance

Avoid splitting between psychotherapy and pharmacotherapy

Avoid or understand splitting between members of staff, either in hospital or in the community

Monitor countertransference feelings
Take into account the patient's ego strength, psychological mindedness, level of intellect and emotional state when deciding whether to use interpretive or supportive techniques. Be prepared to make mistakes and to use a trial-and-error approach. Staff should be aware of their potential lack of responsiveness as a defense against the extreme feelings that these patients engender. Patients are likely to respond very negatively to stereotypical or rigid stances, and staff need to be spontaneous as far as possible, yet keep professional boundaries (not an easy task).

Set boundaries regarding the need for hospitalisation, suicidal behaviour, use of drugs and alcohol, and inappropriate crossing of professional boundaries

Remember that most patients are trying to re-establish their relationship with the rejecting parent by creating a sadomasochistic attachment to staff. Attempts at avoiding such projections, either by defensive countermeasures, trying to prove that the staff are in fact good, or by angry retorts and rejections are likely to lead to disengagement.

"What triggered that extreme reaction?" "How do you think I felt when you attacked me in that way?" "What do you think are the consequences of cutting your wrists?"

These need to be clarified if behaviours threaten the safety of the patient, family, staff or the therapeutic relationship.

Regularly revisit the aims and goals of the therapeutic contract, especially when either the patient or staff have lost their way.

Patients' responses to prescribed medication must form part of the therapeutic interactions and be discussed openly if medication is resisted, sabotaged or abused. Patients may need reminding that the aims of medication are modest: to alleviate the most distressing symptoms and allow time and opportunity to reflect and share feelings.

Recognise that patients may display completely different attitudes, both loving and attacking, within short periods, which can be quite perplexing to staff. Patients are projecting these aspects of themselves onto others, as a way of trying to control these fragmented parts of themselves.

Enable staff to share embarrassing or difficult feelings prompted by their involvement with these patients 
calmly that, although patients are responsible for their behaviour, the staff will be there to do reparative minor surgery if necessary.

\section{Medication approaches}

Although the benefits of medication in borderline personality disorder remain modest, a number of studies have reported improvements when medication is associated with other therapeutic approaches, including cognitive-behavioural and dialectical behavioural therapies, although such psychological techniques are more likely to be used after the patient has been discharged from in-patient care (American Psychiatric Association, 2002). The approach suggested is to treat the three different elements of disturbance in borderline personality disorder with appropriate psychopharmacological management (summarised in Box 6 and discussed more fully below).

For affective dysregulation symptoms, which include mood lability, rejection sensitivity, inappropriate intense anger, depressive 'mood crashes' or outbursts of anger, the initial treatment is with high doses of fluoxetine $(60 \mathrm{mg})$. If this proves to be ineffective, switch to antidepressants that target multiple neurotransmitter systems, such as venlafaxine or monoamine oxidase inhibitors.

When impulse control becomes the predominant feature, characterised by impulsive aggression, selfmutilation or self-damaging binge behaviour (promiscuous sex, substance misuse or reckless spending), selective serotonin reuptake inhibitors (SSRIs) can also be helpful, aided by the addition of lithium, carbamazepine or sodium valproate or low dosage neuroleptics.

Box 6 Medication strategies in borderline personality disorder

- The effect of psychotropic medication is modest: explain this to the patient and family

- Use the rationale of focusing on behavioural problems, mood disturbances or cognitive functioning:

- behavioural (impulsive aggression): SSRIs (fluoxetine up to $60 \mathrm{mg}$ daily, or venlafaxine) or anticonvulsants (carbamazepine, sodium valproate) and lithium

- mood: SSRIs or monoamine oxidase inhibitors

- cognitive: antipsychotics (atypicals); clozapine for self-mutilation

- Avoid alprazolam
Atypical antipsychotics such as olanzapine and risperidone can be added if psychotic features are a problem, and clozapine has also been used in patients exhibiting self-mutilation in the context of psychosis.

Clonazepam can be used if anxiety is high. Avoid alprazolam, which seems to be disinhibitive and to provoke aggressive responses.

\section{Conclusions}

The management of problems presented by patients with personality disorders when admitted to inpatient units needs to be in the therapeutic armamentarium of skilled general psychiatrists and the multidisciplinary staff that work in those units. People with borderline personality disorder present particular challenges to in-patient staff. Although I am not advocating the in-patient unit as the place of choice for the treatment of this disorder, I suggest that the experience on the unit of positive management of the crisis that leads to admission can, in the long term, help these patients by reducing their morbidity and acting-out behaviours. I also believe that these units offer a setting in which evaluation of risk and adequate assessments that might determine treatability can take place, leading to possible referrals to appropriate therapeutic units.

\section{References}

Adshead, G. (2001) Murmurs of discontent: treatment and treatability of personality disorder. Advances in Psychiatric Treatment, 7, 407-415.

American Psychiatric Association (2002) Practice Guidelines for the Treatment of Psychiatric Disorders. Washington, DC: APA.

Bateman, A. \& Fonagy, P. (1999) The effectiveness of partial hospitalisation in the treatment of borderline personality disorder: a randomised controlled trial. American Journal of Psychiatry, 156, 1563-1569.

Bateman, A. \& Tyrer, P. (2002) Effective Management of Personality Disorder. http://www.nimhe.org.uk/ downloads/Bateman_Tyrer.doc

Chiesa, M., Fonagy, P., Holmes, J., et al (2002) Health service utilisation costs by personality disorder following specialist and non-specialist treatment: a comparative study. Journal of Personality Disorders, 16, 160-173.

Davison, S. E. (2002) Principles of managing patients with personality disorder. Advances in Psychiatric Treatment, 8, $1-9$.

Duggan, M. (2002) Developing Services for people with Personality Disorder: The Training Needs of Staff and Services. http://www.nimhe.org.uk/downloads / ReportJuly112002.doc

Fagin, L. (2004) Management of personality disorders in acute in-patient settings. Part 2: Less-common personality disorders. Advances in Psychiatric Treatment, 10, 100-106.

Gabbard, G. O. (2000) Psychodynamic Psychiatry in Clinical Practice. Washington, DC: American Psychiatric Press.

Gallop, R. (1992) Self-destructive and impulsive behaviour in the patient with a borderline personality disorder: rethinking hospital treatment and management. Archives of Psychiatric Nursing, 6, 178-182.

Gunderson, J. (2001) Borderline Personality Disorder: A Clinical Guide. Washington, DC: American Psychiatric Publishing. 
Hendin, H. (1981) Psychotherapy and suicide. American Journal of Psychotherapy, 35, 469-480.

Kaplan, C. A. (1986) The challenge of working with patients diagnosed as having a borderline personality disorder. Nursing Clinics of North America, 21, 429-438.

Kullgren, G. (1988) Factors associated with completed suicide in borderline personality disorder. Journal of Nervous and Mental Diseases, 176, 40-44.

Linehan, M. M., Armstrong, H. E., Suarez, A., et al (1991) Cognitive-behavioural treatment of chronically parasuicida borderline patients. Archives of General Psychiatry, 48, 10601064.

Matsberger, J. (1999) Countertransference in the treatment of the suicidal borderline patient. In Countertransference Issues in Psychiatric Treatment (ed. G. O. Gabbard). Washington, DC: American Psychiatric Press.

Mulder, R. T. (2002) Personality disorder and treatment outcome in major depression: a review. American Journal of Psychiatry, 159, 408-411.

National Institute for Mental Health in England (2003) Personality Disorder: No Longer a Diagnosis of Exclusion. Policy Implementation Guidance for the Development of Services for People with Personality Disorder. London: Department of Health. http://www.nimhe.org.uk/downloads / PDFinal.pdf

Nehls, N. (1994a) Brief hospital treatment plans: innovations in practice and research. Issues in Mental Health Nursing, 15, $1-11$

Nehls, N. (1994b) Brief hospital treatment plans for persons with borderline personality disorder. Perspectives of inpatient psychiatric nurses and community mental health centre clinicians. Archives of Psychiatric Nursing, 8, 303311

Norton, K. \& Dolan, B. (1995) Acting out and the institutional response. Journal of Forensic Psychiatry, 6, 317-332.

Norton, K. \& Hinshelwood, R. D. (1996) Severe personality disorder. Treatment issues and selection for in-patient psychotherapy. British Journal of Psychiatry, 168, 723-731.

O'Brien, L. (1998) Inpatient nursing care of patients with borderline personality disorder. A review of the literature. Australian and New Zealand Journal of Mental Health Nursing, 7, 172-183.

Piccinino, S. (1990) The nursing care challenge: borderline patients. Journal of Psychosocial Nursing, 28, 22-27.

Rouse, J. D. (1994) Borderline and other dramatic personality disorders in the psychiatric emergency service. Psychiatric Annals, 24, 598-602.

Sederer, L. \& Thornbeck, J. (1986) First do no harm: short term inpatient psychotherapy of the borderline patient. Hospital and Community Psychiatry, 37, 692-697.

Stone, M. H. (1990) The Fate of Borderline Patients. New York: Guilford.

Wester, J. M. (1991) Rethinking inpatient treatment of borderline patients. Perspectives in Psychiatric Care, 27, 17-20.

\section{Multiple choice questions}

1 Patients diagnosed with a primary personality disorder:

a should not be admitted

b should be admitted only if they have a comorbid mental disorder

c should be admitted to reduce serious risk to self or others

d who have been charged with committing an offence should be admitted for psychiatric assessment

e should be admitted to provide respite for the family.
2 In-patient care for people with primary personality disorder should be:

a as brief as possible

b used as an opportunity to review the need for medication

c an opportunity to allow patients to understand the source of their problems

$\mathrm{d}$ aimed at containing the crisis that led to the admission

e terminated if the patient continually crosses clearly stipulated behavioural boundaries.

3 Effective in-patient interventions for people with personality disorders will rely on:

a a joint leadership of senior nursing and medical colleagues

b the appointment only of staff who have the personal qualities required to cope with emotional stress

c the existence of local specialist personality disorder services

d the ability to lock the unit when necessary

e close working relationships with community staff.

4 When patients with borderline personality disorder are admitted to hospital:

a suicidal behaviour can be dismissed as a call for help

$b$ elevation of mood is an indication that the crisis has been overcome

c nursing advice that a patient who appears to be testing boundaries should be discharged should always be followed

d families are likely to present obstacles to coherent treatment approaches

e they will require set therapeutic plans following predetermined protocols.

5 When using medication for in-patients with borderline personality disorder, consider that:

a it is likely to undermine psychotherapeutic efforts

$\mathrm{b}$ it is likely to be the mainstay of the treatment approach

c SSRIs are drugs of first choice for patients exhibiting impulsive self-damaging behaviour

d antipsychotics are contraindicated

e mood stabilisers can sometimes be helpful if patient adherence to a treatment regimen is reliable.

\section{MCQ answers}

$\begin{array}{llllllllll}1 & & 2 & & 3 & & 4 & & 5 & \\ \text { a } & \text { F } & \text { a } & \text { T } & \text { a } & \text { T } & \text { a } & \text { F } & \text { a } & \text { F } \\ \text { b F } & \text { b } & \text { T } & \text { b } & \text { F } & \text { b } & \text { T } & \text { b } & \text { F } \\ \text { c } & \text { T } & \text { c } & \text { F } & \text { c } & \text { T } & \text { c } & \text { F } & \text { c } & \text { T } \\ \text { d } & \text { F } & \text { d } & \text { T } & \text { d } & \text { F } & \text { d T } & \text { d F } \\ \text { e } & \text { F } & \text { e } & \text { T } & \text { e } & \text { F } & \text { e } & \text { F } & \text { e } & \text { T }\end{array}$

